\title{
Polygonatum sibiricum F. Delaroche polysaccharide ameliorates HFD-induced mouse obesity via regulation of lipid metabolism and inflammatory response
}

\author{
BO LIU ${ }^{1-3}$, YUAN TANG ${ }^{1}$, ZHENYAN SONG $^{1}$ and JINWEN GE ${ }^{1,4}$ \\ ${ }^{1}$ College of Integrated Traditional Chinese and Western Medicine, Hunan University of Chinese Medicine, Changsha, \\ Hunan 410208; ${ }^{2}$ Central South Food Science Institute of Grain and Oil Co., Ltd., Changsha, Hunan 410100; ${ }^{3}$ Hunan Grain \\ Group Co., Ltd., Changsha, Hunan 410100; ${ }^{4}$ Medical College, Shaoyang University, Shaoyang, Hunan 422000, P.R. China
}

Received September 4, 2020; Accepted February 16, 2021

DOI: $10.3892 / \mathrm{mmr} .2021 .12140$

\begin{abstract}
The present study sought to elucidate the role of Polygonatum sibiricum F. Delaroche polysaccharide (PSP) in high-fat diet (HFD)-induced mouse obesity and investigated the primary molecular mechanism underlaying these effects. An obese mouse model was established by feeding HFD and three doses of PSP were administered intragastrically. Changes in body weight, serum lipids and parameters were recorded and the mechanism was explored by reverse transcription-quantitative PCR and western blotting. Body weight, blood lipids, blood glucose, insulin, resistin, adiponectin, liver weight and abdominal fat pads weight were reduced by PSP and abnormal expression levels of inflammatory factors such as TNF- $\alpha$, IL-6, IL-1 $\beta$ and iNOS and lipid metabolism genes such as FAS, SREBP-1, PPAR $\alpha$ and CPT-1were also reversed by PSP. The 5' adenosine monophosphate-activated protein kinase (AMPK) signaling pathway was activated in PSP mouse liver, leading to lipid-lowering and anti-inflammatory effects. The results therefore suggested that PSP exhibited lipid-lowering and anti-inflammatory effects by activating the AMPK signaling pathway.
\end{abstract}

Correspondence to: Professor Jinwen Ge, College of Integrated Traditional Chinese and Western Medicine, Hunan University of Chinese Medicine, 113 Xueshi Road, Yuelu, Changsha, Hunan 410208, P.R. China

E-mail: 40831556@qq.com

Abbreviations: PSP, Polygonatum sibiricum F. Delaroche polysaccharide; HFD, high-fat diet; TG, triacylglycerol; LDL-C, low-density lipoprotein cholesterol; HDL-C, high-density lipoprotein cholesterol; FFAs, free fatty acids; PVDF, Polyvinylidene fluoride; TBST, Tris-buffered saline containing 0.1\% Tween-20

Key words: obesity, Polygonatum sibiricum polysaccharide, lipid-lowering, 5' adenosine monophosphate-activated protein kinase signaling, inflammation

\section{Introduction}

Obesity, which is defined as a body mass index $>30 \mathrm{~kg} / \mathrm{m}^{2}$, has become one of the major public health problems worldwide and its prevalence has increased in recent years. The World Health Organization reported that the total number of obese individuals is four times the number 30 years ago (1). Obesity and overweight are known to adversely affect human health and the risks and prognosis for a number of serious medical conditions, such as type 2 diabetes and cardiovascular disease, liver diseases, cardiovascular diseases and even cancer $(2,3)$.

Obesity involves pathological changes in a number of tissues, such as adipose tissue and liver. Expanded visceral fat deposits are also linked to dyslipidemia, which is characterized by high triacylglycerol (TG), low-density lipoprotein cholesterol (LDL-C), high-density lipoprotein cholesterol (HDL-C) and insulin resistance, which are often accompanied by impaired metabolic regulation in adipose tissue, leading to an over-production of free fatty acids (FFAs) (4). The flux of FFAs toward the liver results in increased TG deposition and secretion of TG-rich lipoproteins, which in turn affect lipolipase lipase (LPL) activity and the distribution of lipoprotein subtypes (5).

Currently, several drugs, such as sibutramine and orlistat, have been approved by the Food and Drug Administration of the United States, and have been applied for long-term use to prevent obesity (6). Sibutramine increases energy consumption and reduces fat accumulation by inhibiting the re-uptake of monoamine signaling elements (7). Orlistat is a gastrointestinal lipase inhibitor that inhibits intestinal triglyceride absorption (8). However, these drugs inevitably cause side effects such as uneven cardiac rhythm and chest pain, which restrict their clinical application (9).

Polysaccharides are widely distributed in nature: Plants, animals and fungi are valuable sources of polysaccharides. Several types of polysaccharides have been determined to exert multiple functions, such as anti-inflammatory, anti-hyperlipidemic and anti-hypertension effects (10). Among these functions, the lipid-lowering effect of polysaccharides has become a focus of research in recent years. Polysaccharides from soybean (11), flaxseed (12) and Momordica charantia (13) 
have been demonstrated to be effective in treating or preventing obesity.

Polygonatum sibiricum F. Delaroche (P. sibiricum) is a perennial herb of the Asparagaceae family. It is mainly distributed in the Northern Hemisphere, including China, Mongolia, North Korea and the USA (14). P . sibiricum is a well-known traditional medicinal herb in China (15), as well as a health-improving substance (16). P. sibiricum has been widely used in a number of Chinese herbal medicine compounds treating different diseases as recorded in Ben Cao Gang Mu (Compendium of Materia Medica) and its effects in treating diabetes (17), cardiovascular and cerebrovascular diseasess18), somnipathy (19), dyslipidemia and pathological fatigue (20) have also been determined by modern medical methods.

A number of the functional components of $P$. sibiricum have been documented, including polysaccharides, phytosterols, triterpenoids, alkaloids, lignans, flavonoids and phytosterols (21). Among them, polysaccharides and phytosterols are the most abundant medicinal components. $P$. sibiricum polysaccharide (PSP) is mainly composed of galactose, mannose, glucose and galacturonic acid, in molar ratios of 29.63:36.10:15.09:10.20 (22). PSP has been verified to exert a variety of medicinal functions, including anti-inflammatory (23) and anti-tumor effects (24), lowering blood glucose (25), enhancing immunity and promoting the differentiation of bone cells (20).

To investigate if PSP ameliorates high-fat diet (HFD)-induced obesity mice were fed an HFD to establish a high-fat model and PSP was administered intragastrically. The molecular mechanisms were investigated by reverse transcription-quantitative PCR (RT-qPCR) and western blotting.

\section{Materials and methods}

Reagents. PSP (purity 90\%) were obtained from Shaanxi Yikanglong Biotechnology Co., Ltd. Triacylglycerol (TG), total cholesterol (TC), high-density lipoprotein cholesterol (HDL-C) and low-density lipoprotein cholesterol (LDL-C) kits were obtained from Nanjing Jiancheng Bioengineering Institute. The glucometer was from Sannuo Bio-Sensing Technology Co., Ltd., TRIzol ${ }^{\circledR}$ was obtained from Invitrogen; Thermo Fisher Scientific, Inc. and high-capacity cDNA reverse transcription kits were obtained from Applied Biosystems (Thermo Fisher Scientific, Inc.). Antibodies against fatty acid synthase (FAS; cat. no. 4233), phosphorylated (p-) 5' adenosine monophosphate-activated protein kinase (AMPK) $\alpha$ (Thr172; cat. no. 50081), AMPK $\alpha$ (cat. no. 5832), p-acetyl-coenzyme A carboxylase (ACC; Ser79; cat. no. 11818), TNF- $\alpha$ (cat. no. 11948), IL-6 (cat. no. 12912), IL-1 $\beta$ (cat. no. 31202), inducible nitric oxide synthase (iNOS; cat. no. 13120), $\beta$-actin (cat. no. 4970), carnitine palmitoyl transferase I (CPT1; cat. no. 12252) were purchased from Cell Signaling Technology, Inc. The peroxisome proliferator-activated receptor (PPAR $\alpha$; cat. no. ab245119) antibody was purchased from Abcam. The sterol regulatory-element binding protein (SREBP-1; cat. no. PA1-337) antibody and the HRP-conjugated goat anti-rabbit IgG (cat. no. 31460) antibody were obtained from Thermo Fisher Scientific, Inc. RIPA Lysis and Extraction
Buffer was obtained from Beyotime Institute of Biotechnology and protease and phosphatase inhibitors were obtained from Roche Diagnostics. Polyvinylidene fluoride (PVDF) membranes were obtained from Cytiva. An enhanced chemiluminescence (ECL) detection kit was purchased from Pierce (Thermo Fisher Scientific, Inc.).

Experimental animals. A total of 50 eight-week-old C57BL male mice were purchased from Hunan SJA Laboratory Animal Co., Ltd. The mice were housed in a constant temperature and humidity animal room at $23 \pm 2{ }^{\circ} \mathrm{C}$ and a relative humidity of $40-45 \%$, with a 12 -h light/dark cycle, and were acclimated for 7 days. Prior to the animal experiments, the mice were fed a normal diet (Hunan SJA Laboratory Animal Co., Ltd.) and provided water ad libitum. The 50 mice were randomly divided into 5 groups: A normal diet (control) group; a high fat diet (HFD) group and the three PSP groups (mice separately received 200, 400 and $800 \mathrm{mg} / \mathrm{kg}$ of PSP daily by intragastric administration for 3 days before HFD feeding and for the whole experiment). The animal body weights and food intake were recorded weekly. The care and use of the animals and experimental protocols complied with the Guidelines for the Care and Use of Experimental Animals of Hunan University of Chinese Medicine. The project was approved by the Office of Animal Experiment Ethics of Hunan University of Chinese Medicine (approval no. 20190165). At the end of the experiments, the mice were fasted for $12 \mathrm{~h}$ and sacrificed with $\mathrm{CO}_{2}$ for $5 \mathrm{~min}$ as recommended by Pritchett et al (26). The $\mathrm{CO}_{2}$ flow rate was $40 \%$ chamber volume $/ \mathrm{min}$. Blood samples were gathered from the eye for glucose test and biochemical analysis. The liver and epididymal fat pad were immediately removed, weighed, imaged, flash frozen in liquid nitrogen and stored at $-80^{\circ} \mathrm{C}$ for further experiments.

According to the results of body weight changes, the body weights of the 400 and $800 \mathrm{mg} / \mathrm{kg}$ PSP groups were significantly lower compared with the HFD group. However, no significant difference was observed between these two groups. Therefore, the $400 \mathrm{mg} / \mathrm{kg}$ PSP group was chosen for further research.

Biochemical analysis. Blood samples were placed in refrigerator at $4^{\circ} \mathrm{C}$ overnight and then centrifuged at $1,000 \mathrm{x} \mathrm{g}$ for $15 \mathrm{~min}$ at $4^{\circ} \mathrm{C}$. The supernatants were collected as plasma samples. TG, TC, LDL-c and HDL-c levels were measured by commercial assay kits from Nanjing Jiancheng Bioengineering Institute following the manufacturer's instructions. Serum adiponectin (cat. no. MRP300), leptin (cat. no. MOB00B) and resistin (cat. no. MRSN00) levels were measured by ELISAs (R\&D Systems, Inc.), and insulin levels were measured using a mouse insulin-1 ELISA kit (Sigma-Aldrich; Merck KGaA; cat. no. RAB0327).

Histopathological examinations. Samples (epididymal fat pads and liver tissues) were fixed with $10 \%$ neutrally buffered formalin for 2 days at room temperature, dehydrated in an ascending series of alcohol, and then embedded in paraffin by the paraffin embedding machine for $16 \mathrm{~h}$. Paraffin-embedded samples were then cut into $5 \mu \mathrm{m}$ sections. The sections were then deparaffinized with xylene, rehydrated in a descending series of alcohol, rinsed with distilled water for $1 \mathrm{~min}$ and 
Table I. Primers sequences used in reverse transcription-quantitative PCR.

\begin{tabular}{|c|c|c|}
\hline Gene & Forward & Reverse \\
\hline$\beta$-actin & 5'-ATCATGTTTGAGACCTTCACACC-3' & 5'-TAGAGCAACATAGCACAGCTTCTCT \\
\hline $\begin{array}{l}\text { Peroxisome } \\
\text { proliferator-activated } \\
\text { receptor } \alpha\end{array}$ & 5'-TAAAGTACGGTGTGTATGAAGCCAT-3' & 5'-ATGTAGCCTATGTTTAGAAGGCCAG- \\
\hline $\begin{array}{l}\text { Sterol regulatory-element } \\
\text { binding protein-1C }\end{array}$ & 5'-GCCATCGACTACATCCGCTTCTT-3' & 5'-TGGGCTTTGACCTGGCTATCCTC-3' \\
\hline Fatty acid synthase & 5'-AGCACTGCCTTCGGTTCAGTC-3' & 5'-AAGAGCTGTGGAGGCCACTTG-3' \\
\hline $\begin{array}{l}\text { Carnitine } \\
\text { palmitoyltransferase I }\end{array}$ & 5'-CAGCTCGCACATTACAAGGA-3' & 5'-TGCACAAAGTTGCAGGACTC-3' \\
\hline IL-1 $\beta$ & 5'-GCAACTGTTCCTGAACTCAACT-3' & 5'-ATCTTTTGGGGTCCGTCAACT-3' \\
\hline TNF- $\alpha$ & 5'-СССТCACACTCAGATCATCTTCT-3' & 5'-GCTACGACGTGGGCTACAG-3' \\
\hline IL-6 & 5'-TAGTCCTTCCTACCCCAATTTCC-3' & 5'-TTGGTCCTTAGCCACTCCTTC-3' \\
\hline Inducible nitric oxide synthase & 5'-CCTTACGAGGCGAAGAAGGACAG-3' & 5'-CAGTTTGAGAGAGGAGGCTCCG-3' \\
\hline
\end{tabular}

Data are expressed as the mean \pm standard deviation $(\mathrm{n}=6)$. PSP, Polygonatum sibiricum F. Delaroche polysaccharide; HFD, high-fat diet.

stained with hematoxylin (20 min) and eosin (1 min) (H\&E) at room temperature. Images were captured under a light microscope (magnification, x200) in five randomly selected fields of view.

$R N A$ isolation and $R T-q P C R$. Total RNA was isolated from the liver tissues by using RNA extract reagent (TRIzol ${ }^{\circledR}$ ). A total of $2 \mu \mathrm{g}$ of RNA was reverse transcribed into cDNA with cDNA reverse transcription kits (Thermo Fisher Scientific, Inc.) at $42^{\circ} \mathrm{C}$ for $30 \mathrm{~min}$ and then $80^{\circ} \mathrm{C}$ for $10 \mathrm{~min}$ for enzymatic inactivation. qPCR was performed on a CFX96 Real Time PCR system (Applied Biosystems; Thermo Fisher Scientific, Inc.) using the TransStart Green qPCR SuperMix UDG kit (Beijing Transgen Biotech Co., Ltd.; cat. no. AQ111-01). The following thermocycling conditions were used for the qPCR: Initial denaturation at $95^{\circ} \mathrm{C}$ for $1 \mathrm{~min}$; followed by 40 cycles of amplification $\left(94^{\circ} \mathrm{C}\right.$ for $30 \mathrm{sec}, 58^{\circ} \mathrm{C}$ for $25 \mathrm{sec}$ and $72^{\circ} \mathrm{C}$ for $1 \mathrm{~min}$ ), followed by an extension at $72^{\circ} \mathrm{C}$ for $3 \mathrm{~min}$. The primer sequences were described in our previous publication and are shown in Table I (27). The $2^{-\Delta \Delta C q}$ method was used for quantification of the expression levels (28). All these methods were according to the manufacturer's protocols.

Western blot analysis. The liver tissues were homogenized in RIPA Lysis and Extraction Buffer (Beyotime Institute of Biotechnology) on ice for $30 \mathrm{~min}$ and then the suspension was centrifuged at $12,000 \times \mathrm{g}$ for $5 \mathrm{~min}$ at $4^{\circ} \mathrm{C}$. The protein concentration was determined using a BCA Protein assay kit (Abcam). The protein-containing supernatants were equally $(15 \mu \mathrm{g})$ subjected to $10 \%$ SDS-PAGE and then transferred onto a PVDF membrane. The membrane was first blocked with $0.1 \%$ TBS-Tween 20 (TBST) containing 5\% skimmed milk at room temperature for $1 \mathrm{~h}$, followed by incubations with the following primary antibodies: Anti-PPAR $\alpha(1: 2,000)$, anti-SREBP-1C (1:2,000), anti-FAS $(1: 2,000)$, anti-CPT-1 $(1: 2,000)$, anti-IL-1 $\beta(1: 2,000)$, anti-TNF- $\alpha(1: 2,000)$, anti-IL-6 $(1: 2,000)$, anti-iNOS $(1: 2,000)$ and $\beta$-actin $(1: 5,000)$ overnight at $4^{\circ} \mathrm{C}$. Subsequently the membranes were washed in
TBST (3x15 min) and incubated with secondary antibodies $(1: 10,000)$ at room temperature for $1 \mathrm{~h}$. Finally, the membrane was developed with an ECL Plus ${ }^{\mathrm{TM}}$ western blotting detection system. The images were captured using an imaging system (ChemiDoc ${ }^{\mathrm{TM}}$ XRS+, Bio-Rad Laboratories, Inc.). The densitometric analysis was performed using Adobe Photoshop 7.01 (Adobe Systems, Inc.).

Statistical analysis. SPSS v13.0 statistical software (SPSS, Inc.) was used to analyze the data which were expressed as the mean \pm standard deviation. A one-way ANOVA was used for statistical analyses among three groups with the Tukey HSD post hoc test. $\mathrm{P}<0.05$ was considered to indicate a statistically significant difference.

\section{Results}

PSP prevents HFD-induced obesity. To evaluate whether PSP prevents HFD-induced obesity, the mice were administered PSPs as described in the methods section. The body weights of mice in the control group increased steadily up to the end of the experiment. The body weights of the HFD group mice increased quickly from $25.33 \pm 0.44 \mathrm{~g}$ at the beginning to $34.46 \pm 0.89 \mathrm{~g}$ on week 7 and then plateaued until the end of the experiment. The body weights of the different PSP dosages increased more slowly than those of the HFD group, as the $200 \mathrm{mg} / \mathrm{kg}$ PSP group increased from $25.42 \pm 0.46$ to $34.97 \pm 0.88 \mathrm{~g}$, which was lower compared with the HFD group $(\mathrm{P}<0.05)$, while the $400 \mathrm{mg} / \mathrm{kg}$ PSP group was from $25.70 \pm 0.46$ to $33.19 \pm 0.73 \mathrm{~g}$ and the $800 \mathrm{mg} / \mathrm{kg}$ PSP group was from $25.55 \pm 0.48$ to $32.81 \pm 0.71 \mathrm{~g}$, all lower compared with the HFD group $(\mathrm{P}<0.01)$, However, no significant difference was observed between these two groups. Thus, the $400 \mathrm{mg} / \mathrm{kg}$ PSP group was chosen for further experiments and this group was defined as the PSP group hereafter (Fig. 1A).

The average food intake of control group increased steadily and slowly, while the HFD group grew quickly 

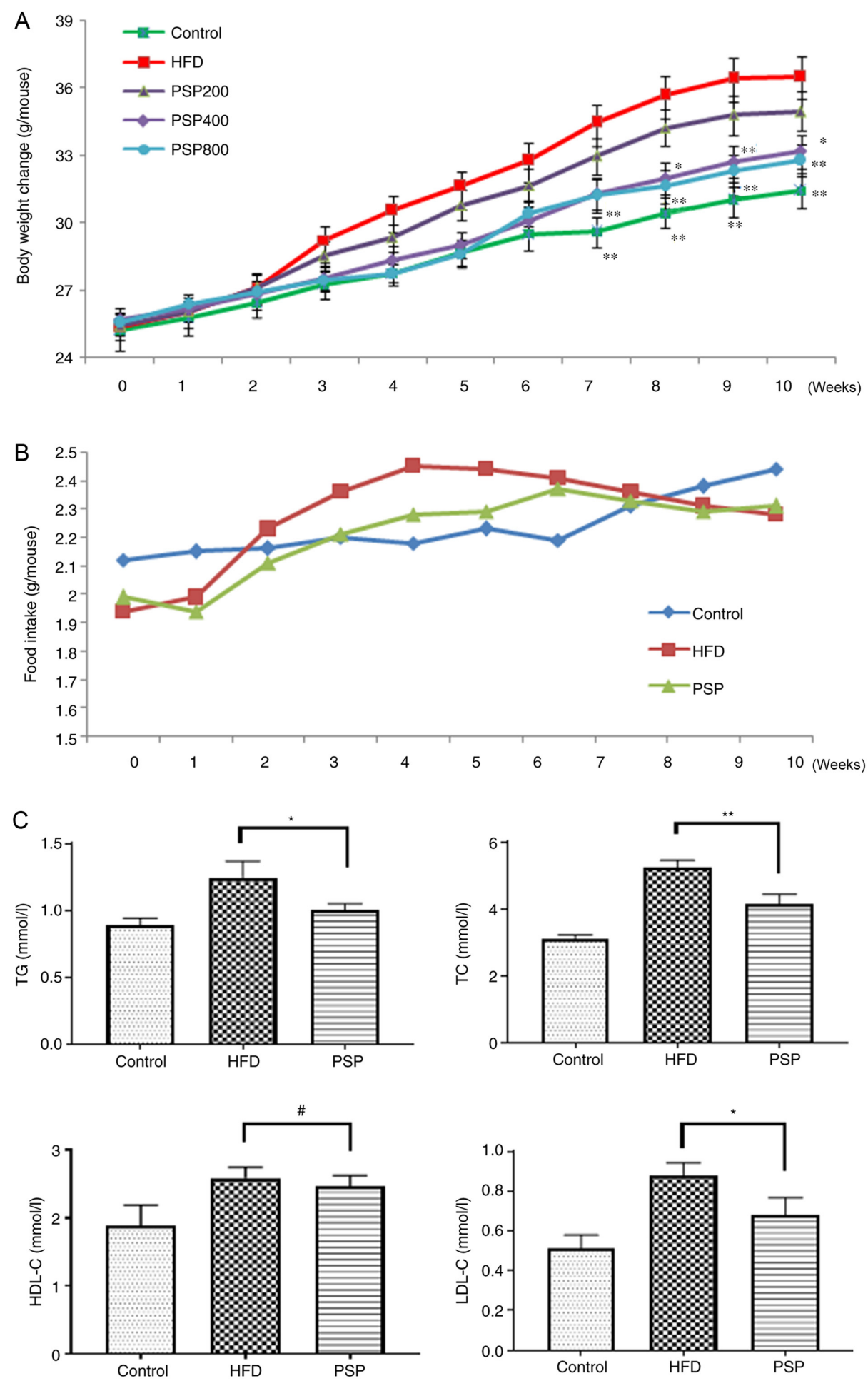

Figure 1. Inhibitory effects of PSP on HFD-induced obesity in mice. C57BL mice were divided into 5 groups. Control group mice received a normal diet, HFD group mice received HFD and three PSP groups mice received HFD and 200, 400 and $800 \mathrm{mg} / \mathrm{kg}$ of PSP by intragastric administration. Body weight and food intake were recorded weekly, Serum lipid index were measured after the mice were sacrificed. (A) Body weight changes and (B) food intake. (C) Serum TG, TC, HDL-C and LDL-C. "P $>0.05,{ }^{*} \mathrm{P}<0.05,{ }^{* *} \mathrm{P}<0.01$. PSP, Polygonatum sibiricum F. Delaroche polysaccharide; HFD, high-fat diet; TG, triacylglycerol; TC, total cholesterol; HDL-C, high-density lipoprotein cholesterol; LDL-C, low-density lipoprotein cholesterol.

in the first 4 weeks and plateaued with a slight decrease toward the end of the experiment at a level that was higher compared with the control group until week 8 . The PSP group mice received less food than the HFD group during weeks 2 and 6 (Fig. 1B). Serum lipid profiles were analyzed using a detection kit. Compared with the levels in the control group, TC, TG, HDL-C and LDL-C were increased following HFD feeding. PSP significantly decreased the levels of TG, TC and LDL-C, while HDL-C was not significantly affected by PSP (Fig. 1C). The serum glucose of the HFD group was $9.62 \pm 0.98 \mathrm{mmol} / \mathrm{l}$, much higher compared with the control group $(5.63 \pm 0.53 \mathrm{mmol} / \mathrm{l})$ while the PSP group was $7.23 \pm 0.55 \mathrm{mmol} / \mathrm{l}$. The insulin concentration was also increased from $0.48 \pm 0.06$ to $0.97 \pm 0.07 \mathrm{ng} / \mathrm{ml}$ by HFD, while 
Table II. Effects of PSP on serum parameters.

\begin{tabular}{lcrrrc}
\hline Parameters & Control & \multicolumn{1}{c}{ HFD } & \multicolumn{1}{c}{ PSP } & P-value; Control vs. HFD & P-value; HFD vs. PSP \\
\hline Glucose $(\mathrm{mmol} / \mathrm{l})$ & $5.63 \pm 0.53$ & $9.62 \pm 0.98$ & $7.23 \pm 0.55$ & $<0.01$ & $<0.01$ \\
Insulin $(\mathrm{ng} / \mathrm{ml})$ & $0.48 \pm 0.06$ & $0.97 \pm 0.07$ & $0.68 \pm 0.09$ & $<0.01$ & $<0.01$ \\
Resistin $(\mathrm{ng} / \mathrm{ml})$ & $0.71 \pm 0.08$ & $2.03 \pm 0.13$ & $1.47 \pm 0.16$ & $<0.01$ & $<0.01$ \\
Leptin $(\mathrm{ng} / \mathrm{ml})$ & $4.07 \pm 0.58$ & $17.05 \pm 2.03$ & $10.34 \pm 0.64$ & $<0.01$ & $<0.01$ \\
Adiponectin $(\mu \mathrm{g} / \mathrm{ml})$ & $6.39 \pm 0.72$ & $4.50 \pm 0.39$ & $5.83 \pm 0.80$ & $<0.01$ & $<0.05$ \\
\hline
\end{tabular}

A

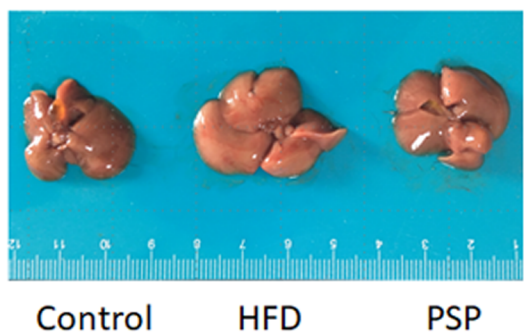

B

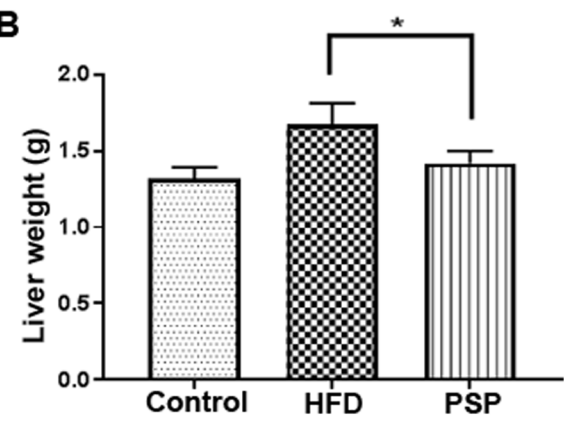

C
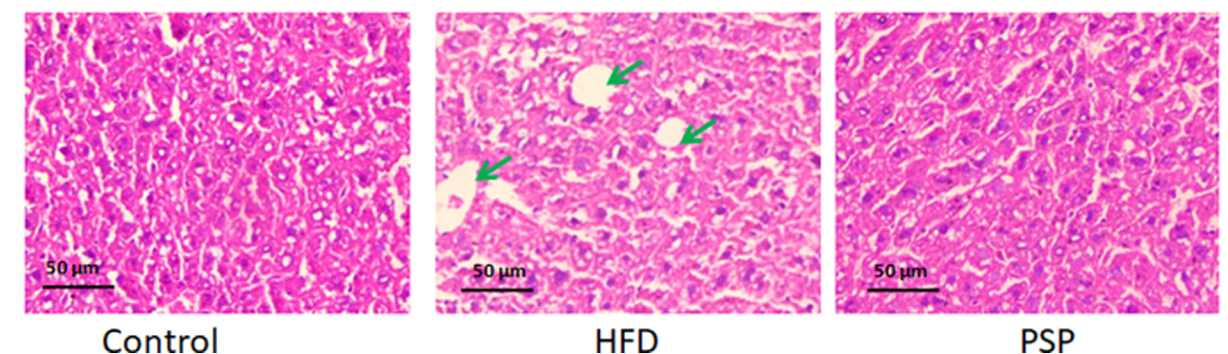

Figure 2. Effect of PSP on relative liver weights and hepatic lipid accumulation in HFD-induced mice. Mice were fed a HFD for 10 weeks and then sacrificed. The livers were removed immediately, weighed, images captured and then stored at $-80^{\circ} \mathrm{C}$. (A) Liver morphology. (B) Liver weight. (C) Liver sections with hematoxylin and eosin staining. Scale bar, $50-\mu \mathrm{m}$. Lipids are indicated by green arrows. ${ }^{*} \mathrm{P}<0.05$. PSP, Polygonatum sibiricum F. Delaroche polysaccharide; HFD, high-fat diet.

the PSP group was only $0.68 \pm 0.09 \mathrm{ng} / \mathrm{ml}$, significantly lower than the HFD group $(\mathrm{P}<0.01$; Table II).

PSP prevents HFD-induced hepatic lesions and adipose hypertrophy. To evaluate the hepatoprotective effects of PSP, mouse livers and abdominal adipose tissue were collected immediately when the mice were sacrificed. The appearance of the livers differed between the groups. As shown in Fig. 2A, the control group livers were in dark red and were the smallest in size. The HFD group livers were large in size and light in color; in addition, fat particles were visible to the naked eye on those livers. The PSP group livers were significantly smaller and the color was darker compared with those of the HFD group. The differences in liver weights were as expected; the liver weight of the control group was $1.32 \pm 0.12 \mathrm{~g}$, while that of the HFD group was $1.67 \pm 0.13 \mathrm{~g}$ and that of the PSP group was $1.41 \pm 0.14 \mathrm{~g}$, which was significantly lower compared with the HFD group (Fig. 2B). The H\&E results revealed why the livers of the HFD group were large and light in color. There was a large amount of fat in the intercellular space of the livers. The PSP group livers contained much less fat (Fig. 2C).

The trend in abdominal adipose tissue was similar to that among the livers; mice in the control group had the smallest adipose tissues weighing $0.80 \pm 0.06 \mathrm{~g}$, while the HFD group was $1.12 \pm 0.13 \mathrm{~g}$ and PSP significantly decreased the weight of the adipose tissues to $0.93 \pm 0.11 \mathrm{~g}$ (Fig. 3A and B). H\&E results indicated that the adipose cells of the HFD group were larger compared with those of the control group. The size of the PSP group adipose cells was significantly decreased (Fig. 3C and D). Adipose secreted factors are an important index of adipose health. The adiponectin in control group was $6.39 \pm 0.72 \mu \mathrm{g} / \mathrm{ml}$, while the HFD decreased it to $4.50 \pm 0.39 \mu \mathrm{g} / \mathrm{ml}$ and PSP effectively reversed this trend to $5.83 \pm 0.80 \mu \mathrm{g} / \mathrm{ml}(\mathrm{P}<0.05)$. In contrast, leptin was increased from $4.07 \pm 0.58$ to $17.05 \pm 2.03 \mathrm{ng} / \mathrm{ml}$ by HFD. Compared with the HFD group, the PSP group exhibited a lower leptin content, only $10.34 \pm 0.64 \mathrm{ng} / \mathrm{ml}(\mathrm{P}<0.01)$. Resistin in the HFD group increased from $0.71 \pm 0.08$ to $2.03 \pm 0.13 \mathrm{ng} / \mathrm{ml}$ and PSP effectively decreased this to $1.47 \pm 0.16 \mathrm{ng} / \mathrm{ml}$ (Table II).

The molecular mechanisms by which PSP prevents $H F D$-induced obesity. The liver is one of the key organs involved in lipid regulation (12). In this present study, mouse livers were collected to explore the potential signaling pathways of PSP in HFD-induced hepatic steatosis. Lipid 
A

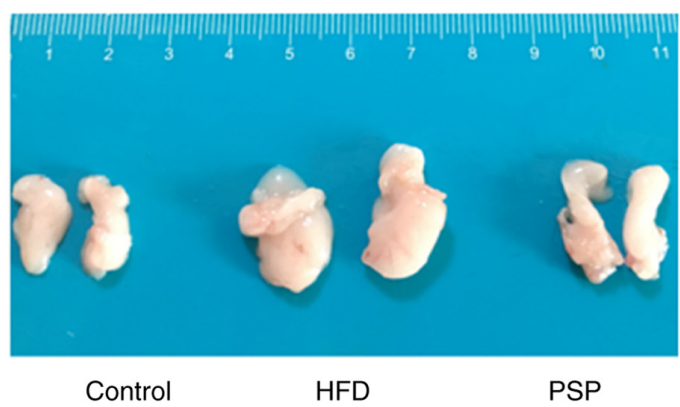

C

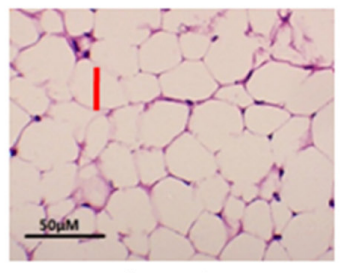

Control

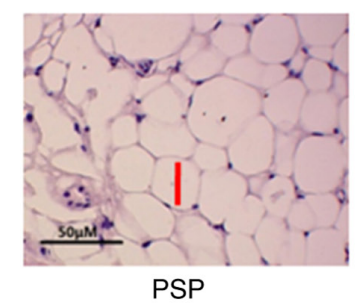

B

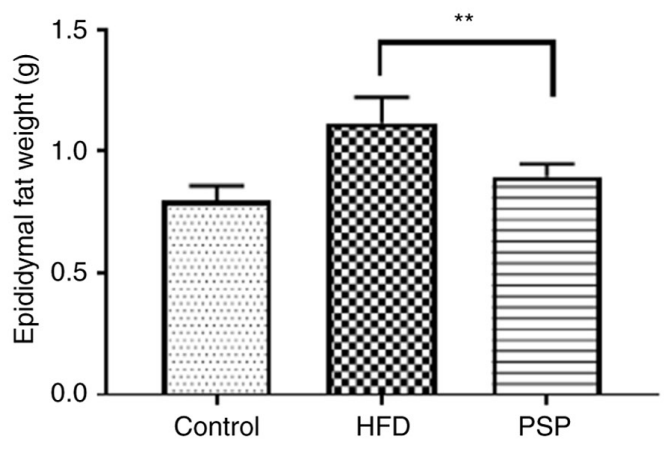

$\mathrm{D}$

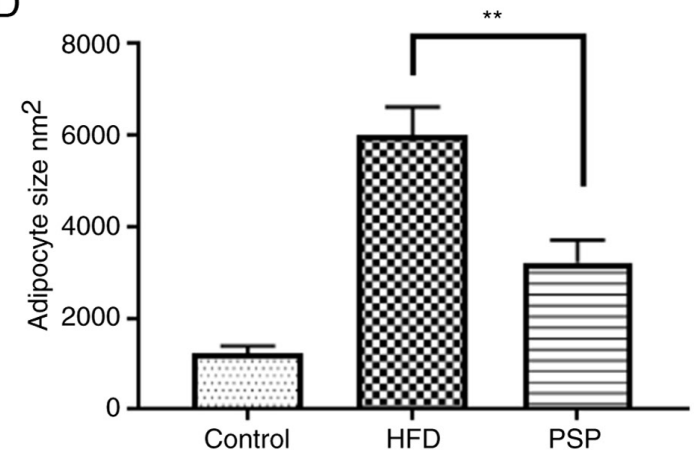

Figure 3. Effects of PSP on adipose tissue and adipocytes of mice administered HFD. The epididymal fat pads were removed immediately, weighed, images captured and then stored at $-80^{\circ} \mathrm{C}$. (A) The morphology of epididymal fat pads. (B) The weight change in the epididymal fat pads. (C) Adipocytes with hematoxylin and eosin staining; scale bar, 50- $\mu \mathrm{m}$. (D) Adipocyte sizes. ${ }^{* *} \mathrm{P}<0.01$. PSP, Polygonatum sibiricum F. Delaroche polysaccharide; HFD, high-fat diet.

metabolic and inflammatory mRNA levels were evaluated by RT-qPCR and western blotting. The fatty acid and cholesterol synthesis-associated genes FAS and SREBP-1 and the inflammatory cytokines TNF- $\alpha$, IL-6, IL-1 $\beta$ and iNOS were highly expressed, while PPAR $\alpha$ and CPT-1 were inhibited in the HFD group. PSP reversed this trend, indicating that PSP inhibits lipid synthesis and inflammation (Figs. 4 and 5A).

The AMPK signaling pathway was assessed and western blotting results indicated that $\mathrm{p}$-AMPK was suppressed in the HFD group, while PSP significantly activated p-AMPK. $\mathrm{P}-\mathrm{ACC}$ results were similar to $\mathrm{p}$-AMPK; clearly blocked in the HFD group and activated in the PSP group (Fig. 5B-D).

\section{Discussion}

Obesity is the commonest chronic metabolic disease in the modern world. Indeed, $>13 \%$ of adults are overweight (29). Obesity is the main cause of a number of serious diseases, including hepatic diseases, diabetes and even cancer (30). The pathogenesis of obesity is very complex. It is widely accepted that the development of obesity is mainly determined by genetic and environmental factors (27). One of the most important environmental factors is the modern lifestyle, which involves the excessive intake of carbohydrates and fat, with a low intake of fiber (31), as well as a lack of physical activity. Although this reason is widely accepted, unhealthy lifestyles remain hard to change. Compared with the effects of lipid-lowering medicines, healthy foods provide long-term protection against obesity and fewer side effects (32). Consequently, research on preventing the pathogenesis of obesity by eating healthy foods has become increasingly popular.

Non-starch polysaccharides are considered effective healthy foods for preventing obesity and one of the main reasons is their unique chemical structures (33). They consist of or contain a number of monosaccharides, such as fructose, galactose, mannose, ribose, rhamnose, xylose and arabinose (34). In addition, the bonds differ from those of starch; for example, oat $\beta$-glucan is $\beta$-1,3/1,4-D-glucan and mushroom and yeast glucans are $\beta-1,3 / 1,6-\mathrm{D}$-glucan (27). These characteristics indicate that polysaccharides are difficult for mammals, which lack the relevant enzymes, to digest and are broken down into monosaccharides, which can be absorbed and used as an energy source (34).

$P$. sibiricum is a traditional Chinese herbal medicine that is effective in treating metabolic diseases such as atherosclerosis and hypertension (31). PSP is one of the main functional components of $P$. sibiricum and has been determined to be effective in enhancing immunity (35) and treating diabetes and diabetic retinal injury (17), acute heart failure (36) and cancer (24). An ethanol extract of $P$. sibiricum is effective in high-fat diet-induced obesity (37). Additionally, PSP regulates bone marrow-derived macrophages to osteoclast differentiation through the ERK/GSK- $3 \beta / \beta$-Catenin signaling pathway (38). However, to the best of the authors' knowledge, there has been no study on the anti-obesity effect of PSP. Thus, the present study evaluated the anti-obesity effect of PSP and 

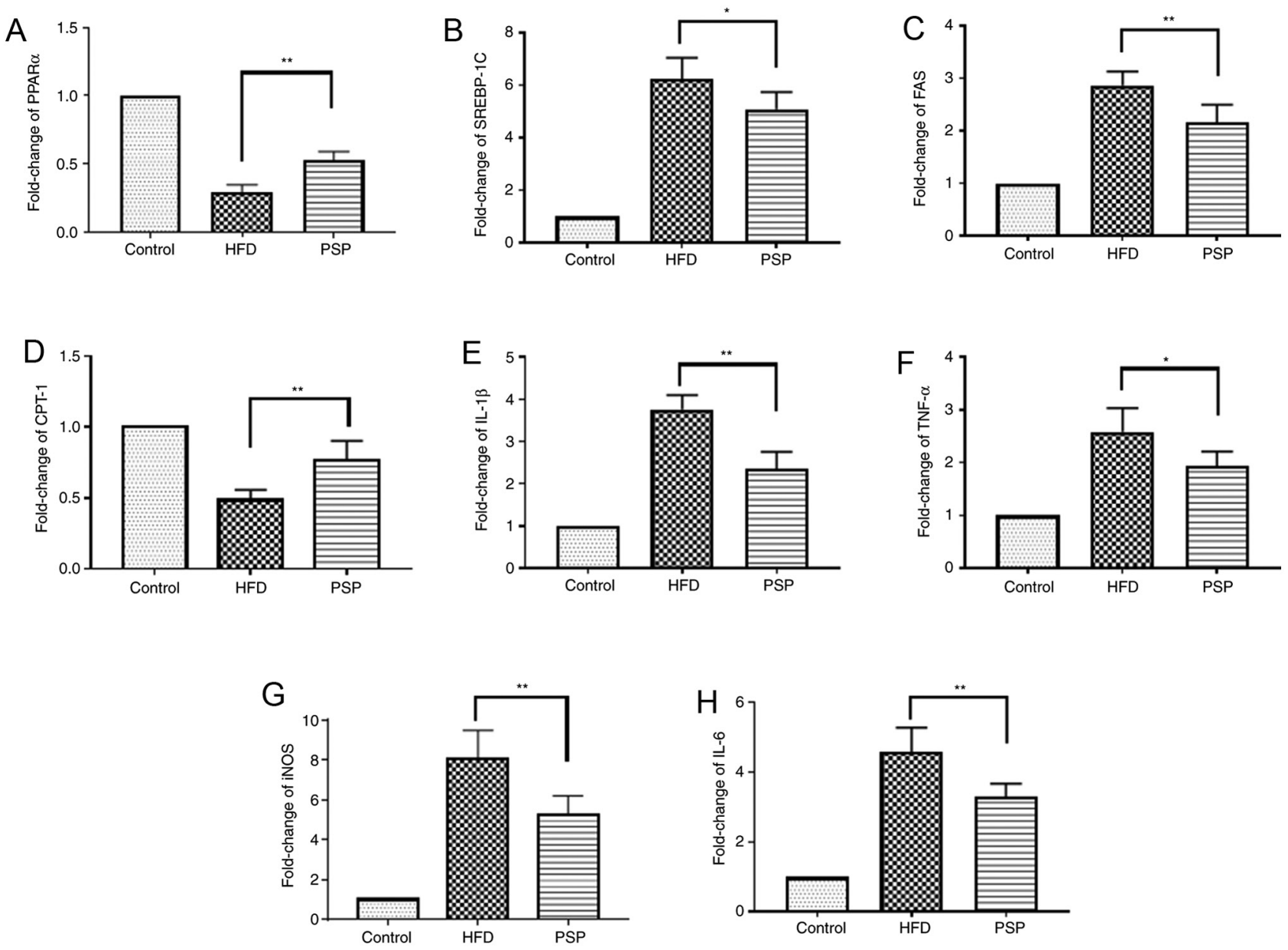

Figure 4. Lipid-associated and inflammatory cytokine mRNA changes. Results of reverse transcription-quantitative PCR. Fold change of lipid-associated (A) PPAR $\alpha$, (B) SREBP-1, (C) FAS and(D) CPT-1 and inflammatory (E) IL-1 $\beta$, (F) TNF- $\alpha$, (G) iNOS and (H) IL-6 mRNA. "P $<0.05$, "* P $<0.01$.PSP, Polygonatum sibiricum F. Delaroche polysaccharide; HFD, high-fat diet; PPAR, peroxisome proliferator-activated receptor; SREBP, sterol regulatory-element binding protein; FAS, fatty acid synthase; CPT-1, carnitine palmitoyltransferase I; iNOS, inducible nitric oxide synthase.

the latent molecular mechanisms. PSP groups mice had slower bodyweight increases and the livers and abdominal fat pads were smaller compared with those of the HFD group. The glucose and insulin contents of the PSP group were lower compared with those of the HFD group. PSP also increased the adiponectin and decreased the leptin and resistin levels. Adiponectin represents one potential contributor to improved insulin sensitivity, which exerts anti-inflammatory and insulin sensitizing effects in the liver and adipocytes (39). Resistin levels are positively associated with increased levels of insulin and glucose and an elevation of resistin levels induces insulin resistance in mice (40). Leptin is another important factor in obesity and inflammation. A reduced leptin level is considered a beneficial effect because leptin has pro-inflammatory effects and contributes to the development of comorbidities of obesity (41). Lipid metabolic and inflammatory cytokines, as well as the AMPK signaling pathway, were also altered by PSP.

AMPK is a serine/threonine kinase and plays critical roles in metabolism (42) and inflammation (43). AMPK activation promotes glucose metabolism and adiposis oxidation which is associated with significant upregulation of autophagy, mitophagy and mitochondrial metabolism. By contrast, it inhibits gluconeogenesis and glycogenesis, fatty acid synthesis and cholesterol synthesis (44). Obesity is associated with chronic energy imbalance and with reduced AMPK activation, accompanied with alterations in glycolysis, hepatic lipid metabolism and inflammation (45).

AMPK downstream genes such as FAS, SREBP-1c, CPT-1 and PPAR- $\alpha$ are associated with lipid biosynthesis and metabolism. An activation of FAS results in lipid synthesis and deposition (44). Conversely, SREBP-1c is negatively correlated with AMPK, meaning that AMPK activity controls SREBP-1c inhibition (46). CPT-1 and PPAR- $\alpha$ are the main directors regulating $\beta$-oxidation which is associated with the hepatic diseases (47). In the present study, AMPK signaling was suppressed in the HFD group and the liver fatty acid and cholesterol synthesis-associated genes FAS and SREBP-1 were highly expressed, while CPT-1 and PPAR- $\alpha$ were inhibited by HFD. PSP significantly activated AMPK signaling, suppressed the expression of FAS and SREBP-1c and activated CPT-1 and PPAR- $\alpha$. HFD-induced obesity is also associated with chronic inflammation (48). In the present study, proinflammatory cytokines, including TNF- $\alpha$, IL-6, IL-1 $\beta$ and iNOS, were highly expressed in the HFD group but significantly inhibited in the PSP group.

In conclusion, the present study is the first, to the best of the authors' knowledge, to investigate the effect of PSP treatment in 


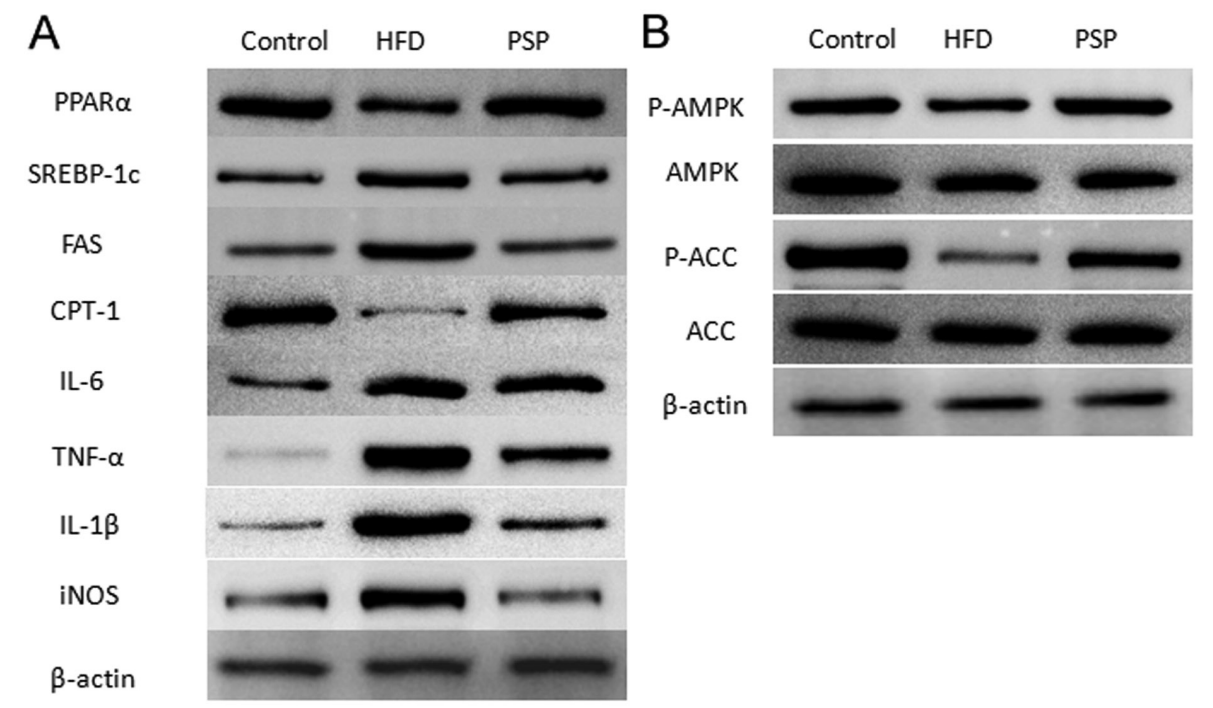

C

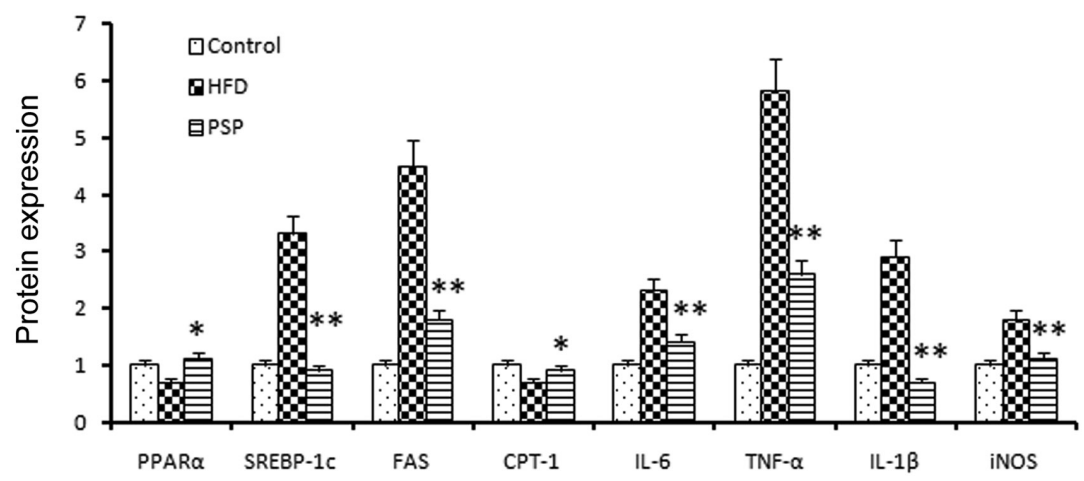

D

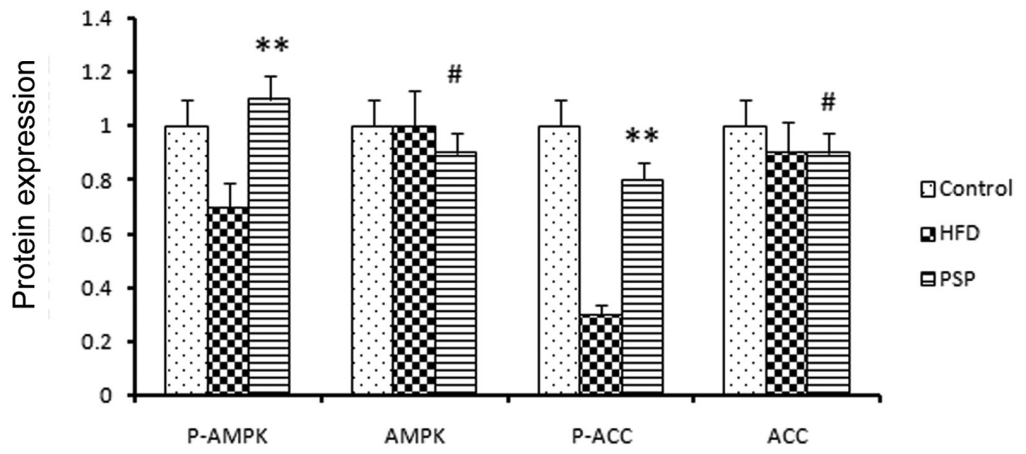

Figure 5. Signal pathway changes and lipid-associated genes and inflammatory cytokine protein changes. (A) Western blotting was performed to reveal the protein expressions of the lipid and inflammatory genes as well as the key genes of the AMPK signal pathway. Lipid synthesis-associated genes and inflammatory genes were inhibited by PSP. (B) P-AMPK and the downstream gene p-ACC was clearly activated by PSP, which demonstrated that the PSP could ameliorate HFD-induced mouse obesity through activating the AMPK signal pathway. (C) Semi-quantification of protein expression levels from part (A) (D) Semi-quantification of protein expression levels from part (B) ${ }^{*} \mathrm{P}>0.05,{ }^{*} \mathrm{P}<0.05,{ }^{* *} \mathrm{P}<0.01$. AMPK, 5 ' adenosine monophosphate-activated protein kinase; p-, phosphorylated; ACC, acetyl-coenzyme A carboxylase; PSP, Polygonatum sibiricum F. Delaroche polysaccharide; HFD, high-fat diet; PPAR, peroxisome proliferator-activated receptor; SREBP, sterol regulatory-element binding protein; FAS, fatty acid synthase; CPT-1, carnitine palmitoyltransferase I; iNOS, inducible nitric oxide synthase.

the prevention of HFD-induced obesity. It is possible that PSP will have the beneficial property of decreasing inflammation in the liver, along with protecting against dyslipidemia, hepatic steatosis and blood glucose increase. It is hypothesized that the mechanism by which PSP supplementation protects against lipid accumulation and inflammatory stress might be due to modulation of the energy balance in the signaling pathway of AMPK.

\section{Acknowledgements}

Not applicable.

\section{Funding}

The present study was supported by the National Natural Science Foundation of China (grant no. 81774174), the Provincial Natural Science Foundation of Hunan (grant no. 2020JJ4345), the China Postdoctoral Foundation (grant no. 2019M652784), the Key Project of the Education Department of Hunan Province (grant no. 18C0400) and the Open Foundation Research Center for Standardization and Functional Engineering Technology of Traditional Chinese Medicine Decoction Pieces of Hunan Province (grant no. 201806). 


\section{Availability of data and materials}

The datasets used and/or analyzed during the current study are available from the corresponding author on reasonable request.

\section{Authors' contributions}

BL and JG designed the experiments and wrote the manuscript; YT performed the animal experiments, histological examination and statistical analysis; BL and ZS performed the RT-qPCR and western blotting experiments. BL and JG confirm the authenticity of all the raw data. JG revised the manuscript and given final approval of the version to be published. All authors read and approved the final manuscript.

\section{Ethics approval and consent to participate}

The care and use of the animals and experimental protocols complied with the Guidelines for the Care and Use of Experimental Animals of Hunan University of Chinese Medicine. The project was approved by the Office of Animal Experiment Ethics of Hunan University of Chinese Medicine (approval no. 20190165).

\section{Patient consent for publication}

Not applicable.

\section{Competing interests}

The authors declare that they have no competing interests.

\section{References}

1. Mishra AK, Dubey V and Ghosh AR: Obesity: An overview of possible role(s) of gut hormones, lipid sensing and gut microbiota. Metabolism 65: 48-65, 2016.

2. Mokdad AH, Ford ES, Bowman BA, Dietz WH, Vinicor F Bales VS and Marks JS: Prevalence of obesity, diabetes, and obesity-related health risk factors, 2001. Jama 289: 76-79, 2003.

3. Van Gaal LF, Mertens IL and De Block CE: Mechanisms linking obesity with cardiovascular disease. Nature 444: 875-880, 2006.

4. Peng CH, Yang MY, Yang YS, Yu CC and Wang CJ: Antrodia cinnamomea prevents obesity, dyslipidemia, and the derived fatty liver via Regulating AMPK and SREBP Signaling. Am J Chin Med 45: 67-83, 2017.

5. Despres JP: Is visceral obesity the cause of the metabolic syndrome? Ann Med 38: 52-63, 2006.

6. Joyal SV: A perspective on the current strategies for the treatment of obesity. Curr Drug Targets CNS Neurol Disord 3: 341-356, 2004.

7. Connoley IP, Liu YL, Frost I, Reckless IP, Heal DJ and Stock MJ: Thermogenic effects of sibutramine and its metabolites. $\mathrm{Br}$ J Pharmacol 126: 1487-1495, 1999.

8. Hsieh CJ, Wang PW, Liu RT, Tung SC, Chien WY, Chen JF, Chen CH, Kuo MC and Hu YH: Orlistat for obesity: Benefits beyond weight loss. Diabetes Res Clin Pract 67: 78-83, 2005

9. Mead E, Atkinson G, Richter B, Metzendorf MI, Baur L, Finer N, Corpeleijn E, O'Malley C and Ells LJ: Drug interventions for the treatment of obesity in children and adolescents. Cochrane Database Syst Rev 11: CD012436, 2016.

10. Nie Y, Lin Q and Luo F: Effects of non-starch polysaccharides on inflammatory bowel disease. Int J Mol Sci 18: 1372, 2017.

11. Lu Y, Zhao A, Wu Y, Zhao Y and Yang X: Soybean soluble polysaccharides enhance bioavailability of genistein and its prevention against obesity and metabolic syndrome of mice with chronic high fat consumption. Food Funct 10: 4153-4165, 2019.
12. Luo J, Qi J, Wang W, Luo Z, Liu L, Zhang G, Zhou Q, Liu J and Peng X: Antiobesity effect of flaxseed polysaccharide via inducing satiety due to leptin resistance removal and promoting lipid metabolism through the AMP-Activated Protein Kinase (AMPK) Signaling Pathway. J Agric Food Chem 67: 7040-7049, 2019.

13. Wen JJ, Gao H, Hu JL, Nie QX, Chen HH, Xiong T, Nie SP and Xie MY: Polysaccharides from fermented Momordica charantia ameliorate obesity in high-fat induced obese rats. Food Funct 10: 448-457, 2019

14. Zhao P, Zhao C, Li X, Gao Q, Huang L, Xiao P and Gao W: The genus Polygonatum: A review of ethnopharmacology, phytochemistry and pharmacology. J Ethnopharmacol 214: 274-291, 2018.

15. Zhang H, Cao Y, Chen L, Wang J, Tian Q, Wang N, Liu Z, Li J, Wang N, Wang X, et al: A polysaccharide from Polygonatum sibiricum attenuates amyloid- $\beta$-induced neurotoxicity in PC12 cells. Carbohydr Polym 117: 879-886, 2015.

16. Zong S, Zeng G, Zou B, Li K, Fang Y, Lu L, Xiao D and Zhang Z: Effects of Polygonatum sibiricum polysaccharide on the osteogenic differentiation of bone mesenchymal stem cells in mice. Int J Clin Exp Pathol 8: 6169-6180, 2015.

17. Wang Y, Lan C, Liao X, Chen D, Song W and Zhang Q: Polygonatum sibiricum polysaccharide potentially attenuates diabetic retinal injury in a diabetic rat model. J Diabetes Investig 10: 915-924, 2019.

18. Kong X, Liu JJ, Li H and Chen ZB: Effect of polysaccharides from Polygonatum sibiricum on lipid-metabolism related mRNA and protein expression in hyperlipidemic mice. Zhongguo Zhong Yao Za Zhi 43: 3740-3747, 2018 (In Chinese).

19. Jo K, Kim H, Choi HS, Lee SS, Bang MH and Suh HJ: Isolation of a sleep-promoting compound from Polygonatum sibiricum rhizome. Food Sci Biotechnol 27: 1833-1842, 2018.

20. Cui X, Wang S, Cao H, Guo H, Li Y, Xu F, Zheng M, Xi X and Han C: A review: The bioactivities and pharmacological applications of polygonatum sibiricum polysaccharides. Molecules 23: 1170,2018

21. Zhao X and Li J: Chemical constituents of the genus Polygonatum and their role in medicinal treatment. Nat Prod Commun 10: 683-688, 2015

22. Sun T, Zhang H, Li Y, Liu Y, Dai W, Fang J, Cao C, Die Y, Liu Q, Wang C, et al: Physicochemical properties and immunological activities of polysaccharides from both crude and wine-processed Polygonatum sibiricum. Int J Biol Macromol 143: 255-264, 2020.

23. Cai J, Zhu Y, Zuo Y, Tong Q, Zhang Z, Yang L, Li X and Yi G: Polygonatum sibiricum polysaccharide alleviates inflammatory cytokines and promotes glucose uptake in high-glucose and high-insulin-induced 3T3L1 adipocytes by promoting Nrf2 expression. Mol Med Rep 20: 3951-3958, 2019.

24. Long T, Liu Z, Shang J, Zhou X, Yu S, Tian H and Bao Y: Polygonatum sibiricum polysaccharides play anti-cancer effect through TLR4-MAPK/NF-kappaB signaling pathways. Int J Biol Macromol 111: 813-821, 2018

25. Wang Y, Qin S, Pen G, Chen D, Han C, Miao C, Lu B, Su C, Feng S, Li W, et al: Original Research: Potential ocular protection and dynamic observation of Polygonatum sibiricum polysaccharide against streptozocin-induced diabetic rats' model. Exp Biol Med (Maywood) 242: 92-101, 2017.

26. Pritchett K, Corrow D, Stockwell J and Smith A: Euthanasia of neonatal mice with carbon dioxide. Comp Med 55: 275-281, 2005.

27. Liu B, Lin Q, Yang T, Zeng L, Shi L, Chen Y and Luo F: Oat $\beta$-glucan ameliorates dextran sulfate sodium (DSS)-induced ulcerative colitis in mice. Food Funct 6: 3454-3463, 2015.

28. Livak KJ and Schmittgen TD: Analysis of relative gene expression data using real-time quantitative PCR and the 2(-Delta Delta C(T)) method. Methods 25: 402-408, 2001.

29. GBD 2015 Obesity Collaborators, Afshin A, Forouzanfar MH, Reitsma MB, Sur P, Estep K, Lee A, Marczak L, Mokdad AH, Moradi-Lakeh M, et al: Health effects of overweight and obesity in 195 Countries over 25 Years. N Engl J Med 377: 13-27, 2017.

30. Santoliquido A, Di Campli C, Miele L, Gabrieli ML, Forgione A, Zocco MA, Lupascu A, Di Giorgio A, Flore R, Pola P, et al: Hepatic steatosis and vascular disease. Eur Rev Med Pharmacol Sci 9: 269-271, 2005.

31. Burgess E, Hassmen P and Pumpa KL: Determinants of adherence to lifestyle intervention in adults with obesity: A systematic review. Clin Obes 7: 123-135, 2017.

32. Liu B, Yang T, Luo Y, Zeng L, Shi L, Wei C, Nie Y, Cheng Y, Lin Q and Luo F: Oat $\beta$-glucan inhibits adipogenesis and hepatic steatosis in high fat diet-induced hyperlipidemic mice via AMPK signaling. J Func Foods 41: 72-82, 2018 
33. Zhang T, Yang Y, Liang Y, Jiao X and Zhao C: Beneficial effect of intestinal fermentation of natural polysaccharides. Nutrients 10 : $1055,2018$.

34. Hervik AK and Svihus B: The role of fiber in energy balance. J Nutr Metab 2019: 4983657, 2019.

35. Yelithao K, Surayot U, Park W, Lee S, Lee DH and You S: Effect of sulfation and partial hydrolysis of polysaccharides from Polygonatum sibiricum on immune-enhancement. Int J Bio Macromol 122: 10-18, 2019.

36. Zhu X, Wu W, Chen X, Yang F, Zhang J and Hou J: Protective effects of Polygonatum sibiricum polysaccharide on acute heart failure in rats 1. Acta Cir Bras 33: 868-878, 2018.

37. Ko JH, Kwon HS, Yoon JM, Yoo JS, Jang HS, Kim JY, Yeon SW and Kang JH: Effects of Polygonatum sibiricum rhizome ethanol extract in high-fat diet-fed mice. Pharm Biol 53: 563-570, 2015.

38. Peng X, He J, Zhao J, Wu Y, Shi X, Du L, Nong M, Zong S and Zeng G: Polygonatum sibiricum polysaccharide promotes

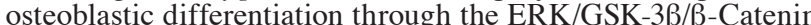
Signaling Pathway In Vitro. Rejuvenation Res 21: 44-52, 2018.

39. Monk JM, Liddle DM, Hutchinson AL, Wu W, Lepp D, Ma DW, Robinson LE and Power KA: Fish oil supplementation to a high-fat diet improves both intestinal health and the systemic obese phenotype. J Nutr Biochem 72: 108216, 2019.

40. Steppan CM, Bailey ST, Bhat S, Brown EJ, Banerjee RR, Wright CM, Patel HR, Ahima RS and Lazar MA: The hormone resistin links obesity to diabetes. Nature 409: 307-312, 2001.

41. Paz-Filho G, Mastronardi C, Franco CB, Wang KB, Wong ML and Licinio J: Leptin: Molecular mechanisms, systemic pro-inflammatory effects, and clinical implications. Arq Bras Endocrinol Metabol 56: 597-607, 2012.
42. Hardie DG: The AMP-activated protein kinase pathway-new players upstream and downstream. J Cell Sci 117 (Pt 23): 5479-5487, 2004.

43. Pan MH, Li MY, Tsai ML, Pan CY, Badmaev V, Ho CT and Lai CS: A mixture of citrus polymethoxyflavones, green tea polyphenols and lychee extracts attenuates adipogenesis in 3T3-L1 adipocytes and obesity-induced adipose inflammation in mice. Food Funct 10: 7667-7677, 2019.

44. Lyons CL and Roche HM: Nutritional modulation of AMPK-impact upon metabolic-Inflammation. Int J Mol Sci 19: 3092, 2018.

45. Garcia D and Shaw RJ: AMPK: Mechanisms of cellular energy sensing and restoration of metabolic balance. Mol Cell 66: 789-800, 2017.

46. Song CY, Shi J, Zeng X, Zhang Y, Xie WF and Chen YX: Sophocarpine alleviates hepatocyte steatosis through activating AMPK signaling pathway. Toxicol In Vitro 27: 1065-1071, 2013.

47. Tailleux A, Wouters K and Staels B: Roles of PPARs in NAFLD: Potential therapeutic targets. Biochim Biophys Acta 1821: 809-818, 2012.

48. Cox AJ, West NP and Cripps AW: Obesity, inflammation, and the gut microbiota. Lancet Diabetes Endocrinol 3: 207-215, 2015.

(i) () $($ This work is licensed under a Creative Commons Attribution-NonCommercial-NoDerivatives 4.0 International (CC BY-NC-ND 4.0) License. 